Original Research

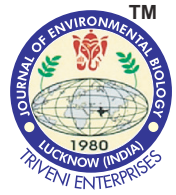

DOI : http://doi.org/10.22438/jeb/39/4/MRN-549

\title{
Resource conservation practices in maize-mustard cropping system: Impact on energy, soil carbon and nutrient dynamics

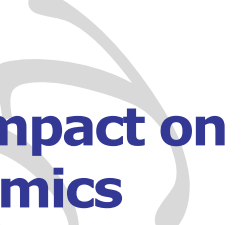

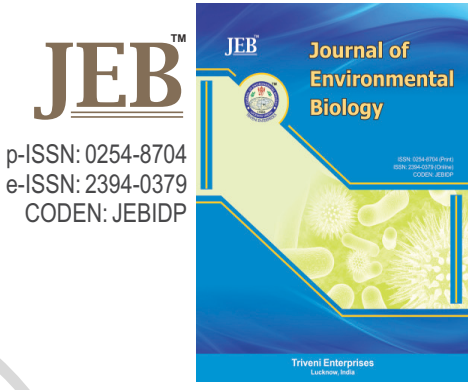

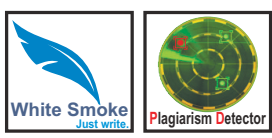

Authors Info

P. Jakhar ${ }^{1,2 *}$, K.S. Rana ${ }^{2}$ A. Dass' ${ }^{2}$ A.K. Choudhary', M. Choudhary ${ }^{3}$, P.P. Adhikary ${ }^{1}$ and J. Maharana ${ }^{4}$

${ }^{1}$ Reserach Centre, ICARIndian Institute of Soil and Water Conservation, Koraput -763 002, India

${ }^{2}$ Division of Agronomy, ICARIndian Agricultural Research Institute, New Delhi-110 012

${ }^{3}$ Division of Crop Production, ICAR-Indian Grassland and Fodder Research Institute, Jhansi- 284 003, India

${ }^{4} \mathrm{KVK}$-Orissa University of Agriculture and Technology, Semiliguda, Koraput -763 002, India

*Corresponding Author Email : icarpraveen@yahoo.co.in

Key words

Carbon sequestration

Energy indices

Hydrogel

Semi-arid region

Zero tillage

Publication Info

Paper received : 24.12.2016

Revised received : 03.06.2017

Accepted :01.11.2017

\section{Abstract}

Aim : Ensuring incessant availability of water and nutrient is an arduous task particularly in rainfed areas. Hence, certain resource conservation measures are needed in those areas for increasing productivity under new emerging cropping systems. Impact of different resource and moisture conservation practices on yield, economics, energy and nutrient dynamics was studied on maize-mustard cropping system in semi-arid region of India.

Methodology : The experiment was laid out in split-plot design with three replications. The main plot treatments consisted of four soil moisture conservation practices in kharif season maize i.e. ridges and furrows (RF), RF + crop residue (CR) @ 4 t ha ${ }^{-1}, \mathrm{RF}+\mathrm{CR} @ 2$ tha ${ }^{-1}+\mathrm{VAM}$ and flat sowing (FS). Five zero tillage (ZT) management techniques in rabi season mustard i.e. $\mathrm{ZT}, \mathrm{ZT}+$ seed priming, ZT +CR@4 tha ${ }^{-1}, \mathrm{ZT}+$ CR @ $2 \mathrm{t} \mathrm{ha}^{-1}+$ Hydrogel and conventional tillage (CT) were undertaken in sub plots in mustard under cropping system mode.

Results : Planting of maize and mustard under RF with 4 tha ${ }^{-1}$ crop residue recorded significantly higher system productivity during 2014-15 and 2015-16 over other treatments. Among the $\mathrm{ZT}$ management practices, $\mathrm{ZT}+\mathrm{CR} 4 \mathrm{t}^{-{ }^{-1}}$ registered maximum MEY in first and second year, respectively. After 2 years of study, maximum SOC sequestration rate (3.48 tha $\left.{ }^{-1} \mathrm{yr}^{-1}\right)$ was observed in RF + CR 2 tha $^{-1}+$ VAM followed by control (FS, 3.40 t ha $^{-1} \mathrm{yr}^{-1}$ ) treatment. Among the sub-plot treatments, ZT + CR 2 tha $^{-1}+$ hydrogel recorded maximum

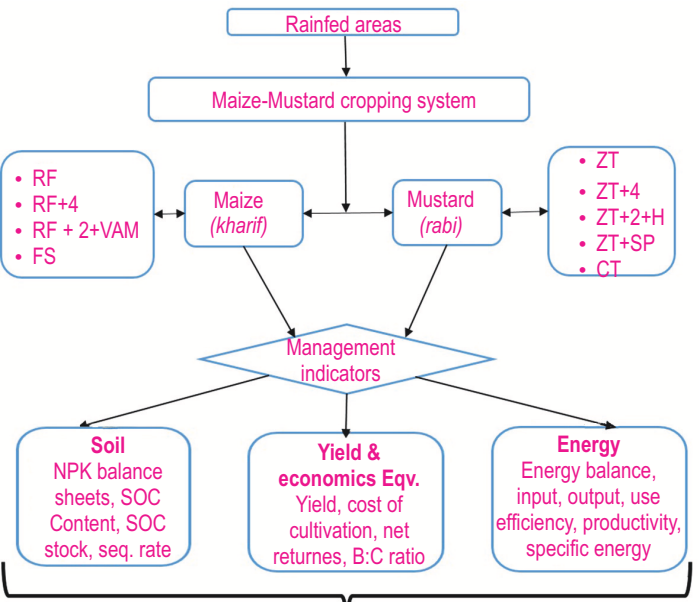

Raising maize under RF + crop residue @ 4 tha th $^{-1}$ and mustard with $\mathrm{ZT}+\mathrm{CR} 4$ tha ${ }^{-1}$ was superior in productivity, net NPK availability, energy productivity and net returns in maize-mustard cropping under rainfed areas.

the tune of $3.42 \mathrm{tha}^{-1} \mathrm{yr}^{-1}$, respectively. Nutrient balance studies showed, RF + CR 4 tha ${ }^{-1}$ having positive $\mathrm{N}$ balance $\left(75 \mathrm{~kg} \mathrm{ha}^{-1}\right)$ whereas under sub-plot treatments $\mathrm{N}$ balance varied from 113-154 kg ha-1 with maximum $\mathrm{N}$ balance obtained in $\mathrm{CT}$.

Interpretation : Raising maize under RF moisture conservation technique with application of crop residue @ $4 \mathrm{t}$ $\mathrm{ha}^{-1}$ and mustard with ZT + CR $4 \mathrm{tha}^{-1}$ were found superior in terms of overall productivity in maize-mustard cropping system, which also fetched maximum net returns. Higher output energy was obtained in above mentioned treatments, while energy-use efficiency and energy productivity was higher under control treatments. 


\section{Introduction}

India possesses about 141.58 million hectare net sown area, out of which $58 \%$ is rainfed. It contributes $40 \%$ to country's food grain production and support $66 \%$ livestock population. Likewise, $40 \%$ of the population depends on rainfed agriculture and its performance is critical to enhance production, achieve and sustain high agricultural growth in years to come (CRIDA, 2015). In rainfed areas across India, maize-mustard cropping system is the promising one. Maize-mustard cropping system is predominant in sub-tropical hill ecosystems of north-eastern region (Munda et al., 1999, Panwar, 2008), south-eastern Rajasthan (Kumpawat, 2004) and Jammu region (Nandan et al., 2013). Both the crops of this system are nutrient-exhaustive and deplete soil nutrients and moisture extensively. Poor recycling of resources leads to emergence of multiple deficiencies in this cropping system (Das et al., 2010).

Conservation tillage and residue retention (mulching) has been found very effective in sustaining rainfed farming (Saha et al., 2010). Management practices such as conservation tillage (Lal et al., 1999) and soil moisture conservation measures can improve the SOC stock and net carbon sink potential which is the core of soil health. Keeping in view the finite carbon sink capacity of soil (Chung et al., 2010), extensively cultivated lands, if put under zero tillage and also other soil moisture conservation measures, can act as potential carbon sinks. Soil management practices like application of manures, fertilizers, irrigation of semiarid and marginal lands for crop production can increase the carbon status (Schlesinger, 2000).

Some work has been done on nutrient management aspect of maize-mustard cropping system but effect of moisture conservation practices and tillage aspects with their residual effects has not been explored enough. Keeping these facts in view the present investigation was carried out with the objective to study the effect of soil moisture enhancing practices and conservation tillage on performance of rainfed maize-mustard cropping system.

\section{Materials and Methods}

Study area : A field experiment was conducted at the Research Farm of ICAR-Indian Agricultural Research Institute, New Delhi during 2014-16. The experimental site was situated at an altitude of $228.6 \mathrm{~m}$ above msl in a semi-arid subtropical climatic belt. The total precipitation received during kharif (monsoon) season of 2014 and 2015 was 395.4 and $710 \mathrm{~mm}$, while for rabi (winter) season it was 227.8 and $22.0 \mathrm{~mm}$ in first and second year, respectively. The soil was sandy loam in texture and slightly alkaline in reaction ( $\mathrm{pH} 7.7)$.

Treatments and layout : The experiment was laid out in split-plot design with three replications. The main plot treatments consisted of four moisture conservation practices in kharifseason maize viz. ridges and furrows $(\mathrm{RF}), \mathrm{RF}+\mathrm{crop}$ residue $(\mathrm{CR}) @ 4 \mathrm{t} \mathrm{ha} \mathrm{-}^{-1}, \mathrm{RF}+$ CR @ $2 \mathrm{t} \mathrm{ha}^{-1}+\mathrm{VAM}$, and flat sowing (FS). Five zero tillage (ZT) management techniques in rabi season mustard viz. ZT, ZT + seed priming, ZT + CR @ 4 t ha ${ }^{-1}, \mathrm{ZT}+\mathrm{CR} @ 2$ t ha $^{-1}+$ Hydrogel and conventional tillage (CT) in sub plots in mustard under cropping system mode.

Cultural operations : Under CT, field was prepared with a disc plough followed by two pass of disc harrow and planking in the last to have a uniform seed bed of fine tilth. No tillage operation was carried out in ZT plot, except mustard sowing. Crop residues of both the crops were applied by spreading the material uniformly on the field just after sowing. Maize variety 'Pusa composite 3 ' and 'Pusa mustard 28' was sown with a spacing of $60 \mathrm{~cm}$. In ZT plots weeds were managed by Glyphosate @ 2.0 I ha ${ }^{-1} 10$ days before sowing and Pendimethalin @ $0.75 \mathrm{~kg}$ a.i. ha ${ }^{-1}$ as preemergence application.

Data and sample analysis : In soil samples, SOC, N, P and K were estimated by the following methods : Walkley and Black (Jackson, 1973), Modified Kjeldahl (Subbiah and Asija, 1956), Olsen et al. (1954) and Flame photometer (Jackson, 1958), respectively. On the basis of primary data (SOC and BD values) SOC stock, build-up rate and sequestration rate was calculated (Lenka et al., 2013). For estimation of energy, inputs and outputs for each item and agronomic practices followed (expressed in MJ ha $^{-1}$ ), energy equivalents were adopted from various available literature (Devasenapathy et al., 2009; Azarpour, 2012). Net energy, energy-use efficiency and energy productivity were calculated as suggested by Mittal and Dhawan (1988) and Mandal et al. (2015). Mean data of two years was statistically analyzed in split-plot design using the technique of analysis of variance with appropriate least significant difference (LSD) value at $5 \%$ level of probability (Gomez and Gomez, 2010).

\section{Results and Discussion}

The productivity of maize-mustard cropping system on the basis of maize grain equivalent yield (MEY) as influenced by different treatments is presented in Table 1. Planting of maize and mustard under RF with 4 t ha $^{-1}$ crop residue (CR) recorded significantly higher MEY (8.43 and $8.01 \mathrm{t} \mathrm{ha}^{-1}$ ) followed by RF + CR $2 \mathrm{t} \mathrm{ha}^{-1}+\operatorname{VAM}$ (7.56 and 7.21 $\mathrm{t} \mathrm{ha}^{-1}$ ) during 2014-15 and 2015-16, respectively. The increment in MEY was recorded as 25.8 and $26.0,11.5$ and $11.0,42.1$ and $37.8 \%$ over RF, RF + CR $2 \mathrm{t} \mathrm{ha}^{-1}$ + VAM and FS during 2014-15 and 2015-16, respectively. Panwar (2008) reported higher MEY under conjugation of organic and inorganic combinations in maize -mustard cropping system. Under sub-plots treatments, ZT+ CR $4 \mathrm{t}^{-1} \mathrm{a}^{-1}$ logged 11.2 and 16.3, 9.4 and 17, 11.5 and $30 \%$ higher MEY over ZT, ZT+ SP and CT in first and second year of experiment, respectively. It is evident that applying 4 tha $^{-1} \mathrm{CR}$ in ZT have supported the soil in terms of developing favourable conditions for plant growth. Similar findings for MEY in maize- 
mustard cropping system was reported by Nandan et al. (2013).

Addition of residues @ 4 tha $^{-1}$ in RF system escalated the cost of cultivation by 4.5 and $4.8 \times 10^{3} ₹ \mathrm{ha}^{-1}$ and addition of residues @ 2 t ha ${ }^{-1}$ with VAM by 3.4 and $3.6 \times 10^{3}$ tha $^{-1}$ in first and second year, respectively (Table 1). Maximum net returns (91.9 and $88.0 \times 10^{3} ₹$ ha $\left.^{-1}\right)$ as well as $B: C$ ratios (2.13 and 1.89) were recorded in $\mathrm{RF}+\mathrm{CR} 4 \mathrm{t} \mathrm{ha}^{-1}$ during both the years of study. Maximum cost incurred in RF+CR 4 tha $^{-1}$ which can be attributed to higher cost of residue, followed by RF+CR $2 \mathrm{t} \mathrm{ha}^{-1}+\mathrm{VAM}$ treatment. Similar findings for economics are also reported by Das et al. (2010) for maize-mustard cropping system in Meghalaya region and Kumpawat (2004) in Bhilwara region of Rajasthan. Under sub-plots, the cost of cultivation increased with increasing levels of crop residues in ZT ( 38.2 and $41.2 \times 10^{3} ₹$ ha $^{-1}$ ) to maximum at $Z T+C R 4$ tha $^{-1}\left(42.7\right.$ and $46.1 \times 10^{3} ₹$ ha $\left.^{-1}\right)$ during 2014-15 and 2015-16, respectively. Maximum net returns to the tune of 79.8 and $83.8 \times 10^{3} ₹$ ha $^{-1}$ were obtained in ZT + CR 4 tha $^{-1}$ which was closely followed by ZT+CR 2 tha $^{-1}+$ hydrogel (78.0 and $77.9 \times 10^{3} ₹ h^{-1}$ ) during first and second year of experiment, respectively, and both were statistically at par.

Energy indices directs that input energy under 2 and 4 tha $^{-1}$ CR was about 1.7 and 2.5 times higher than no-residue (Table 2). Energy-use efficiency under main plot treatment in RF+CR 4 tha ${ }^{-1}$ reduced by $63 \%$ in both the years whereas in sub-plots treatment $\mathrm{ZT}+\mathrm{CR} 4 \mathrm{t} \mathrm{ha}^{-1} 82$ and $64 \%$ in comparison to respective control treatments in first and second year respectively. The reason attributed is that with the application of CR under these treatments very high energy input was added. Higher gross output energy was recorded under RF with $\mathrm{CR} 4 \mathrm{t} \mathrm{ha}^{-1}$ followed by $\mathrm{RF}+\mathrm{CR} 2 \mathrm{t} \mathrm{ha}^{-1}+$
VAM due to higher yields obtained for both maize and mustard crops under these treatments. Significantly higher energy output was obtained in treatment ZT+CR $4 \mathrm{t} \mathrm{ha}^{-1}$ (255 and $267 \mathrm{GJ} \mathrm{ha}^{-1}$ ) followed by ZT+CR 2 tha ${ }^{-1}+$ hydrogel ( 248 and $253 \mathrm{GJ} \mathrm{ha}^{-1}$ ) in first and second year, respectively. Highest value for energy intensiveness ( 3.59 and $3.45 \mathrm{MJ}^{-1}$ ) was observed in RF+CR $4 \mathrm{t}$ ha $^{-1}$. For sub-plots, 4.05 and $3.79 \mathrm{MJ}^{-1}{ }^{-1}$ were obtained for treatment $\mathrm{ZT}+\mathrm{CR} 4$ tha $^{-1}$ and minimum for $\mathrm{CT}$ and $\mathrm{ZT}$ treatments (1.73 and 1.69 MJ $₹^{-1}$ ) during the years 2014-15 and 2015-16, respectively.

Soil organic carbon (SOC) is an important index of soil health vis-à-vis crop yield. In comparison to initial SOC $\left(4.68 \mathrm{~g} \mathrm{~kg}^{-1}\right)$, marginal improvement was observed in all the treatments except for control treatment of both main and sub-plots (Fig.1). Numerically higher and similar SOC values $\left(4.81 \mathrm{~g} \mathrm{~kg}^{-1}\right)$ were observed in RF + CR 4 tha ${ }^{-1}$ and RF + CR 2 tha $^{-1}+$ VAM treatment of moisture conservation practices. Whereas flat sowing (control) showed marginal dip in SOC (4.67 $\left.\mathrm{g} \mathrm{kg}^{-1}\right)$ over the initial values (4.68 $\left.\mathrm{g} \mathrm{kg}^{-1}\right)$. Decomposition rates of soil organic matter is slow under minimal tillage and residue retention practices, therefore $\mathrm{OC}$ content takes longer time to decompose (Gwenzi et al., 2009). In sub-plot treatments, CR application based treatments (ZT + CR $4 \mathrm{t}$ ha $^{-1}$ and ZT + CR 2 tha $^{-1}+$ hydrogel) upheld numerically higher SOC values (4.84 and $4.82 \mathrm{~g} \mathrm{~kg}^{-1}$, respectively) over no-residue treatment. Higher SOC in residue plots than no-residue can be ascribed to quantity of residue (about 16 tha $^{-1}$ in two years) addition and process of carbon sequestration (Abdullah, 2014).

SOC stock was maximum in residue based treatments both in main and sub-plots (Fig. 1). After 2 years of study, SOC

Table 1 : Effect of different treatments on maize equivalent yield (MEY) and cropping system economics

\begin{tabular}{|c|c|c|c|c|c|c|c|c|c|c|}
\hline \multirow{2}{*}{ Treatment } & \multicolumn{2}{|c|}{$\begin{array}{l}\text { MEY } \\
\left(\mathrm{t} \mathrm{ha}^{-1}\right)\end{array}$} & \multicolumn{2}{|c|}{$\begin{array}{l}\text { Cost of cultivation } \\
\left(\times 10^{3} ₹ \mathrm{ha}^{-1}\right)\end{array}$} & \multicolumn{2}{|c|}{$\begin{array}{l}\text { Gross returns } \\
\left(\times 10^{3} ₹ h^{-1}\right)\end{array}$} & \multicolumn{2}{|c|}{$\begin{array}{l}\text { Net returns } \\
\left(\times 10^{3} ₹ \mathrm{ha}^{-1}\right)\end{array}$} & \multicolumn{2}{|c|}{$\begin{array}{l}\text { B:C } \\
\text { Ratio }\end{array}$} \\
\hline & 2014-15 & $2015-16$ & 2014-15 & 2015-16 & 2014-15 & 2015-16 & 2014-15 & 2015-16 & 2014-15 & $2015-16$ \\
\hline \multicolumn{11}{|c|}{$\begin{array}{l}\text { Moisture conservation technicques } \\
\text { (Kharif) }\end{array}$} \\
\hline $\mathrm{RF}$ & 6.70 & 6.36 & 38.7 & 41.8 & 108 & 108 & 69.6 & 66.1 & 1.80 & 1.58 \\
\hline $\mathrm{RF}+\mathrm{CR} 4$ tha $^{-1}$ & 8.43 & 8.01 & 43.2 & 46.6 & 135 & 135 & 91.9 & 88.0 & 2.13 & 1.89 \\
\hline \multicolumn{11}{|l|}{$\mathrm{RF}+\mathrm{CR} 2$ tha $^{-1}$} \\
\hline +VAM & 7.56 & 7.21 & 42.1 & 45.4 & 121 & 121 & 79.0 & 75.3 & 1.88 & 1.66 \\
\hline FS & 5.93 & 5.81 & 38.1 & 41.1 & 96 & 98 & 57.8 & 57.1 & 1.52 & 1.39 \\
\hline $\mathrm{CD}(\mathrm{P}=0.05)$ & 0.49 & 0.43 & - & - & 8.3 & 6.0 & 8.3 & 6.0 & 0.21 & 0.14 \\
\hline \multicolumn{11}{|l|}{ ZT management (Rabi) } \\
\hline ZT & 6.86 & 6.65 & 38.2 & 41.2 & 111 & 112 & 72.4 & 71.0 & 1.88 & 1.71 \\
\hline ZT+ S Priming & 6.97 & 6.61 & 38.5 & 41.5 & 112 & 111 & 73.7 & 69.7 & 1.90 & 1.67 \\
\hline $\mathrm{ZT}+\mathrm{CR} 4$ tha $^{-1}$ & 7.63 & 7.74 & 42.7 & 46.1 & 123 & 130 & 79.8 & 83.8 & 1.86 & 1.82 \\
\hline ZT+ CR2 tha ${ }^{-1}+$ Hydrogel & 7.46 & 7.30 & 41.8 & 45.0 & 120 & 123 & 78.0 & 77.9 & 1.86 & 1.72 \\
\hline CT & 6.84 & 5.94 & 41.3 & 44.7 & 110 & 100 & 68.9 & 55.8 & 1.66 & 1.23 \\
\hline$C D(P=0.05)$ & 0.38 & 0.54 & - & - & 5.1 & 7.6 & 5.1 & 7.6 & 0.12 & 0.17 \\
\hline
\end{tabular}


Table 2 : Effect of different treatments on energy indices of maize-mustard cropping system

\begin{tabular}{|c|c|c|c|c|c|c|c|c|c|c|c|c|c|c|}
\hline \multirow{2}{*}{ Treatment } & \multicolumn{2}{|c|}{$\begin{array}{l}\text { Input } \\
\text { energy } \\
(\text { GJ ha') }\end{array}$} & \multicolumn{2}{|c|}{$\begin{array}{l}\text { Output } \\
\text { energy } \\
\left(\text { GJ ha }{ }^{-1}\right)\end{array}$} & \multicolumn{2}{|c|}{$\begin{array}{l}\text { Energy } \\
\text { balance } \\
\left(\text { GJ ha }{ }^{-1}\right)\end{array}$} & \multicolumn{2}{|c|}{$\begin{array}{l}\text { Energy } \\
\text { use } \\
\text { efficiency }\end{array}$} & \multicolumn{2}{|c|}{$\begin{array}{l}\text { Energy } \\
\text { productivity } \\
\left(\mathrm{kg} \mathrm{GJ}^{-1}\right)\end{array}$} & \multicolumn{2}{|c|}{$\begin{array}{c}\text { Energy } \\
\text { intensiveness } \\
\left(\mathrm{MJ}^{-1}\right)\end{array}$} & \multicolumn{2}{|c|}{$\begin{array}{l}\text { Specific } \\
\text { energy } \\
\left(G J k^{-1}\right)\end{array}$} \\
\hline & $\begin{array}{l}2014- \\
15\end{array}$ & $\begin{array}{l}2015- \\
16\end{array}$ & $\begin{array}{l}2014- \\
15\end{array}$ & $\begin{array}{l}2015- \\
16\end{array}$ & $\begin{array}{l}2014- \\
15\end{array}$ & $\begin{array}{l}2015- \\
16\end{array}$ & $\begin{array}{l}2014- \\
15\end{array}$ & $\begin{array}{l}2015- \\
16\end{array}$ & $\begin{array}{l}2014- \\
15\end{array}$ & $\begin{array}{l}2015- \\
16\end{array}$ & $\begin{array}{l}2014- \\
15\end{array}$ & $\begin{array}{l}2015- \\
16\end{array}$ & $\begin{array}{l}2014- \\
15\end{array}$ & $\begin{array}{l}2015- \\
16\end{array}$ \\
\hline \multicolumn{15}{|l|}{$\begin{array}{l}\text { Moisture cons. tchs. } \\
\text { (Kharif-Maize) }\end{array}$} \\
\hline Ridge and furrows (RF) & 32.9 & 34.1 & 225 & 226 & 192 & 192 & 8.92 & 8.37 & 263 & 234 & 1.68 & 1.60 & 15.6 & 17.8 \\
\hline $\mathrm{RF}+\mathrm{CR} 4$ tha $^{-1}$ & 83.0 & 85.1 & 277 & 276 & 194 & 191 & 3.48 & 3.35 & 107 & 97 & 3.59 & 3.45 & 28.4 & 29.5 \\
\hline $\mathrm{RF}+\mathrm{CR} 2$ tha $^{-1}+\mathrm{VAM}$ & 58.6 & 60.1 & 247 & 245 & 189 & 185 & 4.61 & 4.40 & 140 & 130 & 2.69 & 2.57 & 23.3 & 24.7 \\
\hline Flat sowing & 33.2 & 35.1 & 199 & 203 & 166 & 168 & 7.71 & 7.15 & 229 & 204 & 1.71 & 1.66 & 17.7 & 19.9 \\
\hline $\mathrm{CD}(\mathrm{P}=0.05)$ & - & - & 19.4 & 5.3 & 19.4 & 5.3 & 0.60 & 0.25 & 15.2 & 17.0 & - & - & 1.7 & 1.5 \\
\hline \multicolumn{15}{|l|}{ ZT mgt. (Rabi) } \\
\hline ZT & 36.5 & 38.1 & 228 & 231 & 191 & 193 & 8.00 & 7.76 & 237 & 222 & 1.70 & 1.68 & 14.0 & 14.9 \\
\hline ZT+ S Priming & 36.6 & 38.3 & 229 & 226 & 193 & 188 & 8.08 & 7.48 & 243 & 218 & 1.70 & 1.68 & 13.9 & 15.2 \\
\hline $\mathrm{ZT}+\mathrm{CR} 4$ tha $^{-1}$ & 86.6 & 88.2 & 255 & 267 & 169 & 179 & 3.05 & 3.15 & 92 & 91 & 4.05 & 3.79 & 37.2 & 38.2 \\
\hline $\mathrm{ZT}+\mathrm{CR} 2$ tha $^{-1}+$ Hydrogel & 61.7 & 63.4 & 248 & 253 & 186 & 190 & 4.34 & 4.30 & 131 & 123 & 2.91 & 2.76 & 25.9 & 27.8 \\
\hline CT & 38.5 & 40.2 & 226 & 210 & 188 & 170 & 7.43 & 6.40 & 221 & 176 & 1.73 & 1.69 & 15.1 & 18.6 \\
\hline$C D(P=0.05)$ & - & - & 6.2 & 8.2 & 6.2 & 8.2 & 0.27 & 0.22 & 8.9 & 16.6 & - & - & 1.2 & 2.0 \\
\hline
\end{tabular}

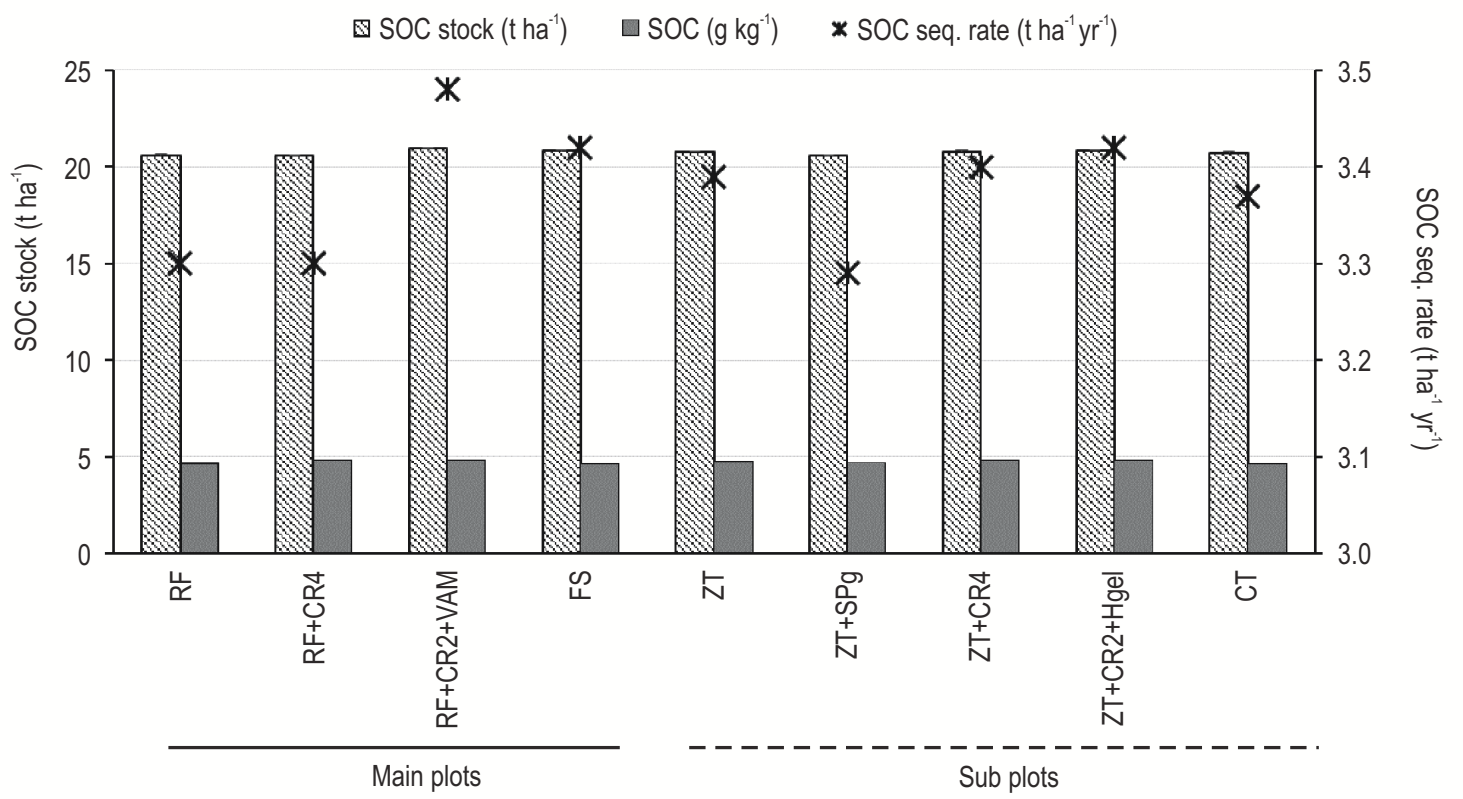

Fig. 1 : Soil organic carbon sequestration as influenced by different treatments

sequestration rate for $30 \mathrm{~cm}$ soil depth varied between 3.29 and $3.48 \mathrm{q} \mathrm{ha}^{-1} \mathrm{yr}^{-1}$ and the maximum value (3.48 $\left.\mathrm{t} \mathrm{ha}^{-1} \mathrm{yr}^{-1}\right)$ was observed in RF + CR 2 t ha $^{-1}+$ VAM followed by $3.40 \mathrm{tha}^{-1} \mathrm{yr}^{-1}$ in control (FS) treatment. Plants are the important sink of carbon and thus they possess high potential for SOC sequestration through improved management practices (Ramesh et al., 2015 and Parmar et al., 2016). Among sub-plot treatments ZT + CR $2 \mathrm{t}$ $\mathrm{ha}^{-1}+$ hydrogel recorded maximum values of SOC sequestration rate to the tune of $3.40 \mathrm{t} \mathrm{ha}^{-1} \mathrm{yr}^{-1}$ and $3.42 \mathrm{t} \mathrm{ha}^{-1} \mathrm{yr}^{-1}$, respectively. West and Post (2002) concluded that a move from CT to ZT (both with residue retention) can sequester an average $48+13 \mathrm{~g} \mathrm{C} / \mathrm{m}^{2}$ year $^{-1}$. 
Table 3 : Nitrogen balance sheet influenced by different treatments in maize-mustard cropping system (at the end of 2 years crop cycle)

\begin{tabular}{|c|c|c|c|c|c|c|c|c|c|}
\hline \multirow{4}{*}{ Treatment } & \multicolumn{4}{|c|}{ Input $\mathbf{N}\left(\mathrm{kg} \mathrm{ha}^{-1}\right)$} & \multicolumn{3}{|c|}{ Output N (kg ha-1) } & \multirow[b]{2}{*}{$\begin{array}{l}\text { Apparent } \\
\text { balance } \\
\left(\mathrm{kg} \mathrm{ha}^{-1}\right)\end{array}$} & \multirow[b]{2}{*}{$\begin{array}{l}\text { Actual } \\
\text { change } \\
\left.\text { (kg ha }^{-1}\right)\end{array}$} \\
\hline & $\begin{array}{l}\text { Initial } \\
\text { availability } \\
\text { in soil }\end{array}$ & $\begin{array}{l}\text { Fertiliser } \\
\text { addition } \\
\#\end{array}$ & $\begin{array}{l}\text { Residue } \\
\text { addition } \\
\# \#\end{array}$ & Total & Uptake & $\begin{array}{l}\text { Final } \\
\text { availability } \\
\text { in soil }\end{array}$ & Total & & \\
\hline & 1 & 2 & 3 & $(1+2+3)$ & 4 & 5 & $(4+5)$ & (IN-OUT) & $(5-1)$ \\
\hline & & & & $=\mathrm{IN}$ & & & $=$ OUT & $=A B$ & $=A C$ \\
\hline \multicolumn{10}{|c|}{$\begin{array}{l}\text { Moisture conservation technicques } \\
\text { (Kharif-Maize) }\end{array}$} \\
\hline Ridge and furrows (RF) & 135 & 400 & 13.1 & 548 & 266 & $136^{\mathrm{bc}}$ & 402 & 146 & 1 \\
\hline$R F+C R 4$ tha $^{-1}$ & 135 & 400 & 33.1 & 568 & 344 & $149^{\mathrm{a}}$ & 493 & 75 & 14 \\
\hline $\mathrm{RF}+\mathrm{CR} 2$ tha $^{-1}+\mathrm{VAM}$ & 135 & 400 & 23.1 & 558 & 304 & $142^{\mathrm{ab}}$ & 446 & 112 & 7 \\
\hline Flat sowing & 135 & 400 & 13.1 & 548 & 240 & $130^{\circ}$ & 369 & 178 & -5 \\
\hline \multicolumn{10}{|c|}{ ZT management (Rabi-Mustard) } \\
\hline Zero tillage $(\mathrm{ZT})$ & 135 & 400 & 7.5 & 543 & 277 & $134^{b}$ & 411 & 132 & -1 \\
\hline $\mathrm{ZT}+$ Seed Priming & 135 & 400 & 7.5 & 543 & 277 & $135^{\mathrm{b}}$ & 413 & 131 & -- \\
\hline $\mathrm{ZT}+\mathrm{CR} 4$ tha $^{-1}$ & 135 & 400 & 51.1 & 586 & 322 & $150^{\mathrm{a}}$ & 472 & 114 & 15 \\
\hline $\mathrm{ZT}+\mathrm{CR} 2$ tha $^{-1}+$ Hydrogel & 135 & 400 & 29.3 & 564 & 306 & $148^{\mathrm{a}}$ & 454 & 113 & 10 \\
\hline Conventional tillage & 135 & 400 & 7.5 & 543 & 260 & $129^{b}$ & 389 & 154 & -6 \\
\hline
\end{tabular}

\#Fertilizer (N) dose: (120+80), for 2 years; \#\#ustard residue (2014) NPK- 0.50:0.15:1.07, Mustard residue (2015) NPK- 0.53:0.16:1.11 and \#\#Maize residue (2014) NPK-0.27:0.12:1.47, Maize residue (2015) NPK-0.29:0.15:1.43

Table 4 : Phosphorus balance sheet as influenced by different treatments in maize-mustard cropping system (at the end of 2 years crop cycle)

\begin{tabular}{|c|c|c|c|c|c|c|c|c|c|}
\hline \multirow{3}{*}{ Treatment } & \multicolumn{4}{|c|}{ Input $P\left(\right.$ kg ha $\left.^{-1}\right)$} & \multicolumn{4}{|c|}{ Output $P\left(\mathrm{~kg} \mathrm{ha}^{-1}\right)$} & \multirow[b]{2}{*}{$\begin{array}{l}\text { Actual } \\
\text { change } \\
\left(\mathrm{kg} \mathrm{ha}^{-1}\right)\end{array}$} \\
\hline & $\begin{array}{l}\text { Initial } \\
\text { availability } \\
\text { in soil }\end{array}$ & $\begin{array}{l}\text { Fertiliser } \\
\text { addition } \\
\#\end{array}$ & $\begin{array}{l}\text { Residue } \\
\text { addition } \\
\# \#\end{array}$ & Total & Uptake & $\begin{array}{l}\text { Final } \\
\text { availability } \\
\text { in soil }\end{array}$ & Total & $\begin{array}{l}\text { Apparent } \\
\text { balance } \\
\left(\mathrm{kg} \mathrm{ha}^{-1}\right)\end{array}$ & \\
\hline & 1 & 2 & 3 & $\begin{array}{l}(1+2+3) \\
=I N\end{array}$ & 4 & 5 & $\begin{array}{l}(4+5) \\
=\text { OUT }\end{array}$ & $\begin{array}{l}\text { (IN-OUT) } \\
=A B\end{array}$ & $\begin{array}{l}(5-1) \\
=A C\end{array}$ \\
\hline \multicolumn{10}{|c|}{$\begin{array}{l}\text { Moisture conservation technicques } \\
\text { (Kharif-Maize) }\end{array}$} \\
\hline Ridge and furrows (RF) & 13.2 & 200 & 5.16 & 218 & 56.3 & $12.9^{b c}$ & 69.2 & 149 & -0.30 \\
\hline $\mathrm{RF}+\mathrm{CR} 4$ tha $^{-1}$ & 13.2 & 200 & 11.2 & 224 & 71.6 & $14.5^{\mathrm{a}}$ & 86.1 & 138 & 1.30 \\
\hline $\mathrm{RF}+\mathrm{CR} 2$ tha $^{-1}+\mathrm{VAM}$ & 13.2 & 200 & 8.16 & 221 & 62.9 & $13.7^{\mathrm{ab}}$ & 76.7 & 145 & 0.50 \\
\hline Flat sowing & 13.2 & 200 & 5.16 & 218 & 48.9 & $12.1^{\mathrm{c}}$ & 60.9 & 157 & -1.10 \\
\hline \multicolumn{10}{|c|}{ ZT management (Rabi-Mustard) } \\
\hline Zero tillage $(\mathrm{ZT})$ & 13.2 & 200 & 2.25 & 215 & 57.2 & $12.5^{b}$ & 69.7 & 146 & -0.70 \\
\hline ZT+ Seed Priming & 13.2 & 200 & 2.25 & 215 & 57.3 & $12.3^{\mathrm{b}}$ & 69.7 & 146 & -0.90 \\
\hline $\mathrm{ZT}+\mathrm{CR} 4$ tha $^{-1}$ & 13.2 & 200 & 19.5 & 233 & 67.3 & $15.6^{\mathrm{a}}$ & 82.9 & 150 & 2.40 \\
\hline $\mathrm{ZT}+\mathrm{CR} 2$ tha $^{-1}+$ Hydrogel & 13.2 & 200 & 10.9 & 224 & 63.9 & $14.5^{\mathrm{a}}$ & 78.4 & 146 & 1.30 \\
\hline Conventional tillage & 13.2 & 200 & 2.25 & 215 & 53.9 & $11.6^{b}$ & 65.5 & 150 & -1.60 \\
\hline
\end{tabular}

${ }^{\#}$ Fertilizer (P) dose: (60+40), for 2 years; ${ }^{\prime \prime}$ Mustard residue (2014) NPK- 0.50:0.15:1.07, Mustard residue (2015) NPK- 0.53:0.16:1.11 and ${ }^{\#}$ Maize residue (2014) NPK-0.27:0.12:1.47, Maize residue (2015) NPK-0.29:0.15:1.43

The nutrient $(\mathrm{N}, \mathrm{P}$ and $\mathrm{K})$ balance sheet of the experiment describes that among different treatments RF $+\mathrm{CR} 4 \mathrm{t} \mathrm{ha}^{-1}$ registered significantly higher available $\mathrm{N}$ values $\left(149 \mathrm{~kg} \mathrm{ha}^{-1}\right)$ which was closely followed by RF+ CR 2tha-1 + VAM $\left(142 \mathrm{~kg} \mathrm{ha}^{-1}\right)$ both being statistically at par (Table 3). Residue based moisture conserving treatments gave 14.6 and $8.5 \%$ higher $\mathrm{N}$ availability in soil as of flat sowing (control). For $\mathrm{N}$ availability under different ZT management practices, ZT + CR 4 tha $^{-1}$ and ZT + CR 2 tha $^{-1}+$ hydrogel yielded statistically at par values (150 and $148 \mathrm{~kg} \mathrm{ha}^{-1}$, respectively). $\mathrm{RF}+\mathrm{CR} 4 \mathrm{t}$ ha ${ }^{-1}$ also showed positive $\mathrm{N}$ balance 
(75 $\left.\mathrm{kg} \mathrm{ha}^{-1}\right)$ whereas FS treatment showed maximum $\mathrm{N}$ balance $\left(178 \mathrm{~kg} \mathrm{ha}^{-1}\right)$. Under sub-plots, ZT + CR 4 tha $^{-1}$ and ZT + CR 2 tha $^{-1}$ + hydrogel gave low $\mathrm{N}$ balance of 114 and $113 \mathrm{~kg} \mathrm{ha}^{-1}$, respectively.

The initial $P$ availability $\left(13.2 \mathrm{~kg} \mathrm{ha}^{-1}\right)$ in the soil was under medium class (10-25 kg ha-1) (Table 4). RF + CR 4 tha $^{-1}$ registered significant superlative at par values to the tune of $14.5 \mathrm{~kg} \mathrm{ha}^{-1}$, which were closely followed by RF+CR $2 \mathrm{t} \mathrm{ha}^{-1}+$ VAM $\left(13.7 \mathrm{~kg} \mathrm{ha}^{-1}\right)$. In comparison to control (FS), an improvement of 6.8, 19.8 and $13.2 \%$ was observed in RF, RF + CR 4 tha $^{-1}$ and RF + CR 2 t ha $^{-1}+$ VAM treatments, respectively. Apparent balance showed that maximum $P$ balance $\left(157 \mathrm{~kg} \mathrm{ha}^{-1}\right)$ in control treatment; whereas residue based superior treatments gave lower values (138 $\mathrm{kg} \mathrm{ha}^{-1}$ for RF+CR4 t ha $\left.{ }^{-1}\right)$. For sub-plots, ZT + CR 4 t ha $^{-1}$ and CT gave statistically at par values (150 $\mathrm{kg} \mathrm{ha}^{-1}$ for each treatment) for apparent $P$ balance. Maximum change $\left(1.30 \mathrm{~kg} \mathrm{ha}^{-1}\right)$ in available $\mathrm{P}$ was observed in $\mathrm{RF}+\mathrm{CR} 4$ tha $^{-1}$ treatment in main plots and $\mathrm{ZT}$ + CR 4 tha $^{-1}$ treatment in subplots.

On a close look at $\mathrm{K}$ balance sheet (Table 5), it can be seen that RF and FS yielded equal values of total $\mathrm{K}$ input $(368 \mathrm{~kg}$ $\mathrm{ha}^{-1}$ ) whereas in sub-plot treatments $\mathrm{ZT}, \mathrm{ZT}+$ seed priming and $\mathrm{CT}$ gave equal $\mathrm{K}$ input values of $336 \mathrm{~kg} \mathrm{ha}^{-1}$. Main plot treatment, RF + CR 4 tha $^{-1}$ registered significantly higher K availability in soil (193 $\left.\mathrm{kg} \mathrm{ha}^{-1}\right)$ which was closely followed by RF + CR 2 tha $^{-1}+$ VAM (187 $\mathrm{kg} \mathrm{ha}^{-1}$ ) but both were statistically at par. In comparison to control, significant improvement of $\mathrm{K}$ availability in soil to the tune of 11.5 and $8 \%$ in RF + CR 4 tha $^{-1}$ and $R F+C R 2$ t ha $^{-1}+V A M$ treatments, respectively was observed. In case of $Z T$ management practices with respect to $\mathrm{K}$ availability; $\mathrm{ZT}+\mathrm{CR} 4$ tha $^{-1}$ registered maximum values (192 $\left.\mathrm{kg} \mathrm{ha}^{-1}\right)$ which were statistically at par to sole ZT (179 $\left.\mathrm{kg} \mathrm{ha}^{-1}\right), \mathrm{ZT}+$ Seed priming (184 kg ha-1) and ZT+CR $2 \mathrm{t} \mathrm{ha}^{-1}+$ Hydrogel $\left(190 \mathrm{~kg} \mathrm{ha}^{-1}\right)$. Maximum positive change $\left(12.5 \mathrm{~kg} \mathrm{ha}^{-1}\right)$ in available $\mathrm{K}$ was observed in $\mathrm{RF}+\mathrm{CR} 4 \mathrm{t} \mathrm{ha}^{-1}$ treatment in main plots, while in sub-plots it was to the tune of $11.4 \mathrm{~kg} \mathrm{ha}^{-1}$ in ZT + CR 4 tha $^{-1}$. Negative Kavailability was observed in case of flat sowing and conventional tillage.

Results indicate that nutrient availability increased with higher dose of crop residue. It proposes that changes in nutrient (NPK) availability due to tillage were limited. Whereas the beneficial effect of crop residue application were more evident because approximately $16 \mathrm{tha}^{-1}$ of residues of maize and mustard was applied in field over a period of two cropping cycles. The higher fertility in RF moisture conservation treatments along with residue brought the synergetic effects of soil water and nutrients on yield of the test crop (Gebrekidan and Uloro, 2015). Large quantities of uptake by crops, remains in the residues even after harvesting, contribute in high nutrient availability (Du Preez and Bennie, 1991). Negative values in non-residue and nonmoisture conservation treatments in actual change and NPK availability indicate the inherent depletion of macronutrient of the soil.

Table 5 : Potassium balance sheet influenced by different treatments in maize-mustard cropping system (at the end of 2 years crop cycle)

\begin{tabular}{|c|c|c|c|c|c|c|c|c|c|}
\hline \multirow{3}{*}{ Treatment } & \multicolumn{3}{|c|}{ Input K (kg ha $\left.{ }^{-1}\right)$} & \multicolumn{5}{|c|}{ Output K (kg ha-1) } & \multirow[b]{2}{*}{$\begin{array}{l}\text { Actual } \\
\text { change } \\
\left(\mathrm{kg} \mathrm{ha}^{-1}\right)\end{array}$} \\
\hline & $\begin{array}{l}\text { Initial } \\
\text { availability } \\
\text { in soil }\end{array}$ & $\begin{array}{l}\text { Fertiliser } \\
\text { addition } \\
\#\end{array}$ & $\begin{array}{l}\text { Residue } \\
\text { addition } \\
\text { \#\# }\end{array}$ & Total & Uptake & $\begin{array}{l}\text { Final } \\
\text { availability } \\
\text { in soil }\end{array}$ & Total & $\begin{array}{l}\text { Apparent } \\
\text { balance } \\
\left(\mathrm{kg} \mathrm{ha}^{-1}\right)\end{array}$ & \\
\hline & 1 & 2 & 3 & $\begin{array}{l}(1+2+3) \\
=I N\end{array}$ & 4 & 5 & $\begin{array}{l}(4+5) \\
=\text { OUT }\end{array}$ & $\begin{array}{l}(\text { IN-OUT) } \\
=A B\end{array}$ & $\begin{array}{l}(5-1) \\
=A C\end{array}$ \\
\hline \multicolumn{10}{|c|}{$\begin{array}{l}\text { Moisture conservation technicques } \\
\text { (Kharif-Maize) }\end{array}$} \\
\hline Ridge and furrows (RF) & 180 & 140 & 48 & 368 & 222 & $177^{\mathrm{bc}}$ & 398 & -30 & -3.4 \\
\hline $\mathrm{RF}+\mathrm{CR} 4$ tha $^{-1}$ & 180 & 140 & 91 & 411 & 292 & $193^{\mathrm{a}}$ & 485 & -73 & 12.5 \\
\hline $\mathrm{RF}+\mathrm{CR} 2$ tha $^{-1}+\mathrm{VAM}$ & 180 & 140 & 70 & 390 & 255 & $187^{\mathrm{ab}}$ & 442 & -52 & 7.3 \\
\hline Flat sowing & 180 & 140 & 48 & 368 & 192 & $173^{c}$ & 365 & 3 & -7.3 \\
\hline \multicolumn{10}{|c|}{ ZT management (Rabi-Mustard) } \\
\hline Zero tillage $(\mathrm{ZT})$ & 180 & 140 & 16 & 336 & 227 & $179^{\mathrm{ab}}$ & 406 & -70 & -0.8 \\
\hline $\mathrm{ZT}+$ Seed Priming & 180 & 140 & 16 & 336 & 225 & $184^{a}$ & 409 & -73 & 3.7 \\
\hline ZT+ CR4tha ${ }^{-1}$ & 180 & 140 & 176 & 497 & 275 & $192^{\mathrm{a}}$ & 467 & 30 & 11.4 \\
\hline $\mathrm{ZT}+\mathrm{CR} 2$ tha $^{-1}+$ Hydrogel & 180 & 140 & 96 & 416 & 260 & $190^{\mathrm{a}}$ & 450 & -33 & 9.5 \\
\hline Conventional tillage & 180 & 140 & 16 & 336 & 213 & $168^{b}$ & 381 & -45 & -12.5 \\
\hline
\end{tabular}

${ }^{\#}$ Fertilizer (K) dose: (40+30), for 2 years; "\#Mustard residue (2014) NPK- 0.50:0.15:1.07, Mustard residue (2015) NPK- 0.53:0.16:1.11 and "\#Maize residue (2014) NPK-0.27:0.12:1.47, Maize residue (2015) NPK-0.29:0.15:1.43 
In conclusion, raising maize under RF moisture conservation technique with application of crop residue @ 4 tha $^{-1}$ and mustard with ZT + CR 4 t ha $^{-1}$ were found superior in terms of productivity in maize-mustard cropping system under rainfed condition fetched maximum net returns. Soil physical and chemical properties were favourably influenced under residue cover than no residue. This cropping system can be remunerative in monetary as well as soil health terms with use of crop residue and conservation based tillage.

\section{References}

Abdullah, A.S.: Minimum tillage and residue management increase soil water content, soil organic matter and canola seed yield and seed oil content in the semi-arid areas of Northern Iraq. Soil Tillage Res., 144, 150-55 (2014).

Azarpour, E.: Determination of energy balance and energy indices in wheat production under watered farming in north of Iran. ARPN J Agri. Bio. Sci., 7, 250-255 (2012).

Chung, H., K.J., Ngo, A.F., Plante and J. Six: Evidence for carbon saturation in a highly structured and organic matter rich soil. Soil Sci. Soc. Am. J., 74, 130-138 (2010).

CRIDA. Vision 2050. ICAR-Central Research Institute for Dryland Agriculture, Santoshnagar, Hyderabad, p. 1 (2015).

Das, A., D. P., Patel, G. C. Munda and P. K. Ghosh: Effect of organic and inorganic sources of nutrients on yield, nutrient uptake and soil fertility of maize (Zea mays)-mustard (Brassica campestris) cropping system. Ind. J. Agri. Sci., 80, 85-88 (2010).

Devasenapathy, P., G. Senthilkumar and P.M. Shanmugam: Energy management in crop production. Indian J. Agron., 54, 80-90 (2009).

Du Preez, C.C. and A.T.P. Bennie: Concentration, accumulation and uptake rate of macro-nutrients by winter wheat under irrigation. $S$. Afr. J. Plant Soil, 8, 31-37 (1991).

Gebrekidan, H. and Y. Uloro: Yield response of maize (Zea mays L.) to tied ridges and planting methods on Entisols and Vertisols of the eastern Ethiopian highlands. In abstracts: Tropical Agriculture. The University of West Indies at St. Augustine, Trinidad and Tobago 88 (2015).

Gomez, K.A. and A. A. Gomez: Statistical procedures for agricultural research, $2^{\text {nd }} E d n$., John Wiley and Sons, New York, p.704 (2010).

Gwenzi, W., J., Gotosa, S. Chakanetsa and Z. Mutema: Effects of tillage systems on soil organic carbon dynamics, structural stability and crop yields in irrigated wheat (Triticum aestivum L.)-cotton (Gossypium hirsutum L.) rotation in semi-arid Zimbabwe. Nut. Cyc. Agroecosys., 83, 211-221 (2009).

Jackson, M.L.: Soil chemical analysis, Asian Publication House, New Delhi, pp. 173-195(1958).

Jackson, M.L.: Soil chemical analysis. Prentice Hall Inc., Englewood, Cliffs, U.S.A., pp. 159-174 (1973).

Kumpawat, B.S.: Integrated nutrient management in maize mustard cropping system in southern Rajasthan. Indian J. Agron., 49, 18-21 (2004).

Lal, R., R.F. Follett, J. Kimble and C.V. Cole: Managing US cropland to sequester carbon in soil. J. Soil Water Conserv., 54, 374-381 (1999).

Lenka, N. K., S., Sudhishri, A., Dass, P. R., Choudhury, S., Lenka and U.S. Patnaik: Soil carbon sequestration as affected by slope aspect under restoration treatments of a degradedalfisol in the Indian sub-tropics. Geoderma, 204,102-110 (2013).

Mandal, S., A. Roy, G.I. Das, R. Ramkrushna, B.C. Lal, A.K. Verma, R.K. Singh, and J. Layek: Energy efficiency and economics of rice cultivation systems under subtropical Eastern Himalaya. En. Sus. Devlop., 28, 115-121 (2015).

Mittal, J.P. and K. C. Dhawan: Research manual on energy requirements in agricultural sector. ICAR, New Delhi, p. 150 (1988).

Munda, G.C., U. K. Hazarika, D.C. Saxena, R. Singh and D.P. Patel: Performance of cropping system under mid altitude rainfed dry terrace of Meghalaya. Ind. J. Hill Farm., 12, 106-10 (1999).

Nandan, B., B. C. Sharma and A. Kumar: Effects of mustard-maize intercropping system on productivity of maize in moisture deficit sub-tropical areas of Jammu and Kashmir. Schol. J. Agri. Sci., 3, 66-72 (2013).

Olsen, S.R., C.L., Cole, F.S. Watanabe and L.A. Dean: Estimation of available phosphorus in soil by extraction with sodium bicarbonate. USDACircular No. 939, Washington, pp.72-75(1954).

Panwar, A.S.: Effect of integrated nutrient management in Maize (Zeamays) - Mustard cropping system (Brasicca campestrasis var. toria) cropping system in mid hills altitude. Ind. J. Agri. Sci., 78, 27-31 (2008).

Parmar, D.K., D. R. Thakur, R.S. Jamwal and Arpana: Effect of long term organic manure application on soil properties, carbon sequestration, soil - plant carbon stock and productivity under two vegetable production systems in Himachal Pradesh. J. Env. Bio., 37, 333-39 (2016).

Ramesh, T., K. M. Manjaiah, K. P. Mohopatra, K. Rajasekar and S.V. Nagchan: Assessment of soil organic carbon stocks and fractions under different agroforestry systems in subtropical hill agro ecosystems of north-east India. Agrof.Sys., Print ISSN 0167-4366 Online ISSN 1572-9680 (2015).

Saha, S., D. Chakraborty, A. R. Sharma, R. K. Tomar, S. Bhadraray, U. Sen, U. K. Behera, T. J. Purakayastha, R. N. Garg and N. Kalra: Effect of tillage and residue management on soil physical properties and crop productivity in maize (Zea mays)-Indian mustard (Brassica juncea) system. Indian J. Agri. Sci., 80, 679-85 (2010).

Schlesinger, W.H.: Carbon sequestration in soils: Some cautions amidst optimism. Agric. Ecosys. Environ., 82, 121-27 (2000).

Subbiah, B.V. and G.L.Asija: A rapid procedure for the estimation of available nitrogen in soils. Curr. Sci.,25, 259-60 (1956).

West, T.O. and W. M. Post: Soil organic carbon sequestration rates by tillage and crop rotation. Soil Sci. Soc. Am. J., 66, 1930-46 (2002). 